\title{
Bedside Ultrasound in the Neonatal Intensive Care Unit: A Wave of the Future
}

\section{Alan M Fujii* and Bharati Sinha}

Department of Pediatrics, Boston Medical Center, Boston University, Boston, MA, USA

Neonatology is a relatively new, age and size determined subspeciality involved in the care acute care of sick and premature newborns. Neonates are in transition from the aquatic, intrauterine environment to an aerobic, pulmonary dependent, environment. The interaction between pulmonary and cardiovascular systems in ill neonates is more complex than at any other time in the spectrum of life. Conventional evaluation of disturbances in these interactions by oxygen saturation, heart rate, respiratory rate, and physical examination are inadequate. Bedside Ultrasound Imaging (BUSI) in the Neonatal Intensive Care Unit (NICU) provides a rapid, non-invasive, real-time window into disturbances in these cardiopulmonary interactions. Traditionally, in the NICU, control of the ultrasound is managed by experts in pediatric cardiology and radiology. Whether the neonatologists themselves should perform 'point of care' ultrasound, is controversial. In reality, 24 hour presence of specialists to provide ultrasound services in the NICU is not achievable. Overall, use of ultrasound in the NICU by neonatologists has lagged behind other specialists in adult intensive care, emergency room medicine and obstetrics where use of ultrasound by the primary care giver is routine. The purpose of this editorial is to discuss the usefulness of BUSI in enhancing clinical care, indications for usage, barriers to implementation and development of training programs that teach use of BUSI in the NICU.

There are four major areas of the body accessible to ultrasound directed evaluation: head and neck, chest, abdomen, and periphery. Cranial, abdominal and peripheral ultrasounds have well-established standard views and standard Doppler interrogation. While premature infants are smaller, more fragile and at least as ill as their adult counterparts, premature infants have less subcutaneous fat and muscle, less distance to the area of interest, and are prime candidates for use of high frequency, high resolution transducers. Premature infants may be more amenable to ultrasonographic evaluation than adult patients. Neonatologists in many parts of the world routinely perform head ultrasound to screen for intraventricular hemorrhage, hydrocephalus and periventricular leukomalacia in preterm babies. Chest and airway ultrasound is currently gaining popularity in adult ICU settings. In an adult ICU population lung ultrasound had a diagnostic accuracy of 92$100 \%$ in identifying pneumothorax, consolidation, interstitial air leak syndrome, and pleural effusion, which was far superior to bedside CXR, when compared with thoracic CT scan [1]. In children with suspected vocal cord palsy, who underwent both laryngoscopy and laryngeal ultrasound, the concordance rate of laryngeal ultrasound was as high as $89.5 \%$ in patients above 12 months age [2]. Clinicians have taken this a step further by using ultrasound in assessment and management of airway [3]. We have used ultrasonographic imaging in neonates to diagnose unilateral diaphragmatic paralysis, pleural effusion, unilateral vocal cord paralysis and a posterior thoracic mass in neonates.

The greatest indication for use of real time ultrasound in the NICU by neonatologists is echocardiography. The poor correlation of cardiac hemodynamics with clinical findings in premature newborns is well known [4]. Neonatal cardiorespiratory evaluation, however, is a moving target with the cardiopulmonary changes from placental oxygenation to pulmonary oxygenation, complicated by superimposed pathology. A simple evaluation of an infant with oliguria may include a BUSI evaluation for left ventricular preload/ volume depletion, poor cardiac function, pulmonary hypertension, coarctation of the aorta, renal abnormalities, distended bladder from outlet obstruction, ascites, necrotizing enterocolitis or sepsis. BUSI, by crossing conventional lines of discipline may provide the intensivist with a global evaluation of an ill neonate that may not be available except by obtaining multiple consults leading to delay in diagnosis and management. BUSI may take some of the guesswork out of clinical medicine.

Introduction of new technology to a specialty practice is always met with roadblocks. How does one organize training and overcome the barriers to implementation? After a brief focused training comprising of didactic course and hands-on sessions, intensive-care unit physicians without previous knowledge of ultrasonography can competently perform basic general ultrasonic examinations looking for chest and abdominal pathology [5]. Many neonatologists have acquired skill in echocardiography by informal, self-directed learning [6]. Organized courses run by pediatric cardiologists and neonatologists are gaining popularity in UK, Australia and New Zealand. Other methods used are informal self directed learning using books and multimedia personal computer-based programs, sometimes enhanced by direct supervision by experienced echocardiographer. The recent practice guideline and consensus statement for targeted echocardiography in the NICU by the Writing Group of the American Society of Echocardiography in collaboration with the European Association of Echocardiography and the Association for European Pediatric Cardiologists [7] was written by experts from the US and Europe to provide a structure to teach neonatologists functional echocardiography. At the Boston Medical Center in Boston, Massachusetts, two neonatologists with experience in echocardiography currently perform bedside echocardiography to monitor cardiac function, evaluate murmurs, hemodynamic assessment of patent ductus arteriosus and check central line position. Echocardiography digital images obtained by neonatologists are uploaded on to a central server and read remotely by a pediatric cardiologist. All initial targeted echocardiograms are followed by a detailed full echocardiogram performed by the echo technician and evaluated by a pediatric cardiologist. We believe that this model can be used by other neonatologists who have acquired prior training to audit their own echocardiography practice while at same time providing 24 hour echo-tech availability to the NICU.

As with any investigative tool, BUSI should be combined with, rather than replace clinical acumen. Implementation of BUSI by neonatologists would need significant resources including a dedicated ultrasound machine for NICU use, training and monitoring of

*Corresponding author: Alan M Fujii, MD, Department of Pediatrics, Boston Medical Center, Boston University, Boston, USA, Tel: 617-414-3735; Fax: 617414-7297; E-mail: Alan.Fujii@bmc.org

Received March 20, 2012; Accepted March 22, 2012; Published March 24, 2012

Citation: Fujii AM, Sinha B (2012) Bedside Ultrasound in the Neonatal Intensive Care Unit: A Wave of the Future. J Pulmonar Respirat Med 2:e112. doi:10.4172/2161-105X.1000e112

Copyright: ( 2012 Fujii AM, et al. This is an open-access article distributed under the terms of the Creative Commons Attribution License, which permits unrestricted use, distribution, and reproduction in any medium, provided the original author and source are credited. 
appropriate use by newly trained staff and close collaboration with specialist services. Logistic challenges with study reporting and image archiving should also be addressed. Well designed studies to prove that BUSI impacts clinical outcome are needed before it gains wider use. Formal guidelines governing the use and monitoring of BUSI performed by neonatologists in the NICU will refine practice in the NICU, minimize interpretational errors, and allow patient care to proceed in the presence of a safety net. There is no more dedicated investigator than the physician involved in direct clinical care seeking to do the best for the patient.

\section{References}

1. Xirouchaki N, Magkanas E, Vaporidi K, Kondili E, Plataki M, et al. (2011) Lung ultrasound in critically ill patients: comparison bedside chest with radiography. Intensive Care Med 37: 1488-1493.

2. Vats A, Worley GA, de Bruyn R, Porter H, Albert DM, et al. (2004) Laryngeal ultrasound to assess vocal fold paralysis in children. J Laryngol Otol 118: 429-431.
3. Kristensen MS (2011) Ultrasonography in the management of the airway. Acta Anaesthesiol Scand 55: 1155-1173.

4. Osborn DA, Evans N, Kluckow M (2004) Clinical detection of low upper body blood flow in very premature infants using blood pressure, capillary refill time, and central-peripheral temperature difference. Arch Dis Child Fetal Neonatal Ed 89: 168-173.

5. Chalumeau-Lemoine L, Baudel JL, Das V, Arrive L, Noblinski B, et al (2009) Results of short-term training of naive physicians in focused general ultrasonography in an intensive-care unit. Intensive Care Med 35: 1767-1771.

6. Evans N (2000) Echocardiography on neonatal intensive care units in Australia and New Zealand. J Paediatr Child Health 36: 169-171.

7. Mertens L, Seri I, Marek J, Arlettaz R, Barker P, et al. (2011) Targeted Neonata Echocardiography in the Neonatal Intensive Care Unit: practice guidelines and recommendations for training. Writing Group of the American Society of Echocardiography (ASE) in collaboration with the European Association of Echocardiography (EAE) and the Association for European Pediatric Cardiologists (AEPC). J Am Soc Echocardiogr 24: 1057-1078. 